\title{
Precision measurement of the top quark mass with single top events at CMS
}

\author{
Mintu Kumar ${ }^{a, *}$ \\ ${ }^{a}$ Tata Institute of fundamental research, \\ Homi bhabha road, Mumbai, India \\ E-mail: mintu.kumar@cern.ch
}

A measurement of the top quark mass in single top events produced in the $t$-channel at the CMS experiment is presented. The $t$-channel is the dominant process for single top quark production at the LHC. The $t$-channel process provides a unique phase space with different color reconnection conditions than that of $t \bar{t}$ events, which are are exploited for the most precise measurements of the top-quark mass. The final state comprises a single top quark along with a light quark giving rise to at least two jets, (one of which arises from the hadronization of a b quark), an isolated high-momentum lepton (electron or muon), and large missing transverse momentum due to an escaping neutrino from the $\mathrm{W}$ boson decay. The study is based on proton-proton collision data, equivalent to $35.9 \mathrm{fb}^{-1}$ integrated luminosity, recorded at $\sqrt{s}=13 \mathrm{TeV}$ by the CMS experiment during 2016. Dominant standard model backgrounds are studied in different kinematic regions depending on the number of b-tagged quarks and light-flavor jets in the final state. A multivariate technique relying on boosted decision trees is employed to optimally separate the signal from backgrounds. The top quark mass is reconstructed using kinematic information of the final-state objects, namely the charged lepton, missing transverse momentum, and jets. We obtain the top quark mass $m_{\mathrm{t}}=172.13_{-0.70}^{+0.69} \mathrm{GeV}$, by fitting the reconstructed mass distribution using an appropriate combination of parametric shapes.

\footnotetext{
*** The European Physical Society Conference on High Energy Physics (EPS-HEP2021), ***

*** 26-30 July $2021 * * *$

*** Online conference, jointly organized by Universität Hamburg and the research center DESY ***
}

\footnotetext{
${ }^{*}$ Speaker
} 


\section{Introduction}

A precise measurement of the top quark mass is of profound importance, both for theory and experiment, being an important parameter of the standard model (SM). It constitutes a major input to verify the self-consistency of the SM. Among all elementary particles, it is the largest contributor in terms of radiative corrections to the mass of the Higgs boson. Most of the top-quark mass measurements to date have been performed with $\mathfrak{t t}^{\mathrm{t}}$ events at the Large Hadron Collider (LHC) at CERN. Thanks to the partially independent phase-space compared to t' production, the single top $t$-channel offers a suitable alternative to measure the top quark mass. The $t$-channel diagram shown in Fig. 1 constitutes the dominant process for single top quark production in pp collisions at the LHC, with a total cross-section of $216.99_{-7.71}^{+9.04} \mathrm{pb}$ calculated at next-to-leading order in quantum chromodynamics (QCD).
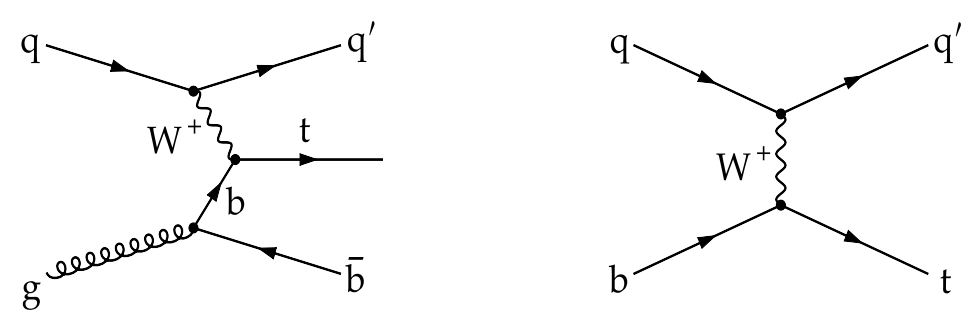

Figure 1: Feynman diagrams of $t$-channel single top quark production at leading order corresponding to four- (left) and five-flavor (right) schemes.

\section{Event reconstruction and categorization}

In this analysis, candidate events are selected by requiring at least one isolated muon or electron and a large missing momentum due to the escaping neutrino. Exactly two jets are required, of which one must be b-tagged. The event categories are defined according to the number of total jets and b-tagged jets $(n \mathrm{~J} m \mathrm{~T})$. The $2 \mathrm{~J} 1 \mathrm{~T}$ category is the most significant contributor to $t$-channel signal events and is used for the measurement of the top quark mass. The four-momentum of the top quark (and hence its mass) is reconstructed from the fourvectors of its decay products: the charged lepton, the neutrino, and the b-tagged jet.

QCD multijet production has a tiny acceptance in the phase space used in the analysis. A sideband (SB) in data enriched in QCD multijet events are defined by inverting the isolation (identification) criteria for the selected muon (electron). QCD templates are derived by subtracting the total non-QCD contribution in this SB. The QCD contribution in the signal region is then estimated by means of a two-component binned maximum-likelihood fit to the distribution of the transverse mass of the charged lepton plus neutrino system $\left(m_{\mathrm{T}}\right)$ as shown in Fig 2. The method is validated in the QCD-dominated 2J0T category. As such, for selecting signal events, we require the $m_{\mathrm{T}}$ value to be greater than $50 \mathrm{GeV}$ in order to suppress the QCD contribution. 

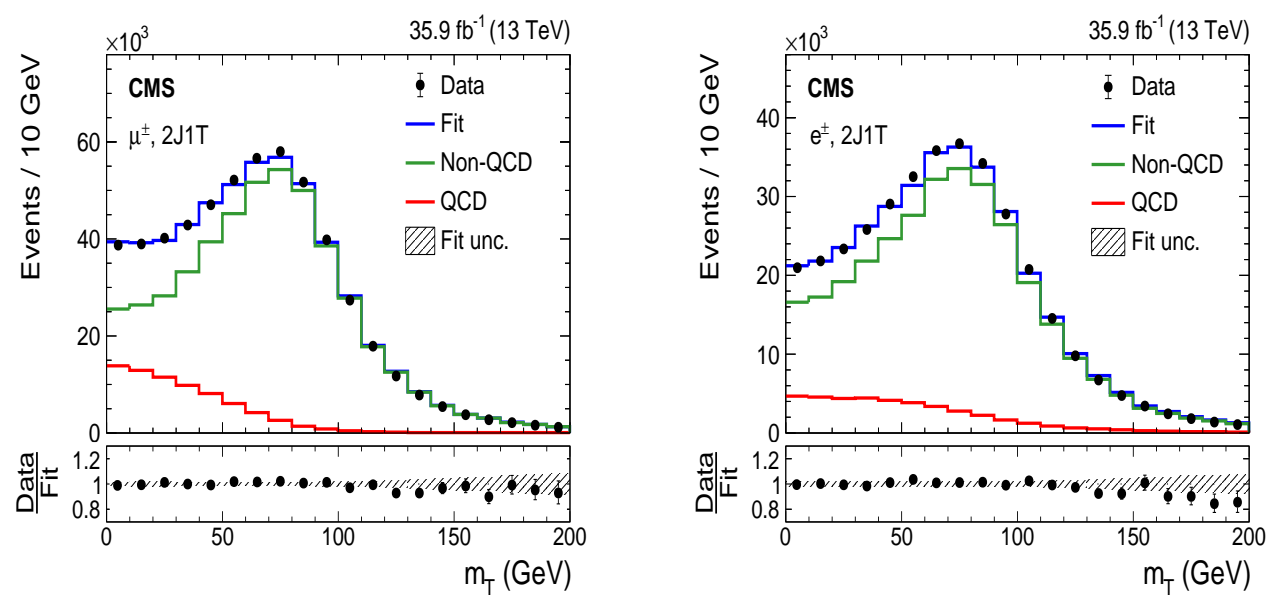

Figure 2: Postfit distributions of $m_{\mathrm{T}}$ for the muon (left) and electron (right) final state.

\section{Fit strategy and results}

A number of kinematic variables are combined into a multivariate analysis (MVA) discriminator to optimally separate $t$-channel single top quark events from SM backgrounds. These variables are selected such that they have a significant power to distinguish signal and background. One boosted decision tree $(\mathrm{BDT})$ is designed per lepton flavor. The BDT responses have $\approx 13 \%$ correction with the reconstructed top quark mass. A criterion on the BDT response $>0.8$ is chosen, which results in $64 \%$ (58\%) signal purity for a $20 \%$ efficiency for the muon (electron) final state.

The $y=\ln \left(m_{\mathrm{t}}\right)$ distributions obtained from the muon and electron final states are considered in a simultaneous maximum-likelihood fit. The QCD multijet contribution is subtracted from data before the fit with the residual distribution described by a parametric 1D model, as:

$$
\mathrm{F}\left(y ; y_{0}, \mathrm{f}_{t-\mathrm{ch}}, \mathrm{f}_{\text {Top }}, \mathrm{f}_{\text {EWK }}\right)=\mathrm{f}_{t-\text { ch }} \cdot \mathrm{F}_{t-\mathrm{ch}}\left(y ; y_{0}\right)+\mathrm{f}_{\text {Top }} \cdot \mathrm{F}_{\text {Top }}\left(y ; y_{0}\right)+\mathrm{f}_{\text {EWK }} \cdot \mathrm{F}_{\text {EWK }}(y)
$$

Here, $\mathrm{F}_{t-\mathrm{ch}}, \mathrm{F}_{\mathrm{Top}}$, and $\mathrm{F}_{\mathrm{EWK}}$ represent the parametric shapes for the signal, top quark, and electroweak backgrounds, respectively. The parameter $y_{0}$ of the combined signal and top quark background template is the parameter of interest. $\mathrm{F}_{t-\mathrm{ch}}$ is modeled with a sum of an asymmetric Gaussian core, and a Landau tail function. $\mathrm{F}_{\text {Top }}$ is described by a Crystal Ball function and $\mathrm{F}_{\mathrm{EWK}}$ is modeled with a Novosibirsk function. The top quark mass is obtained from the post fit $y$ distribution as shown in Fig. 3 by taking the exponential of the post fit value of $y_{0}$. The signal and background normalizations are profiled (prof) by including them as nuisance parameters in the fit. These normalization nuisance parameters are constrained using log-normal priors with 15\%, 6\%, and $10 \%$ based on their respective cross-section uncertainties. All other sources of uncertainty are externalized. Uncertainties are calculated from the difference between the offset-corrected postfit values of $m_{\mathrm{t}}$ corresponding to the nominal and varied templates. The largest shift relative to the nominal result is quoted as the uncertainty. The dominant source of uncertainties (in a descending order) are jet energy scale, signal modeling, b-quark hadronization model, and color reconnection. 

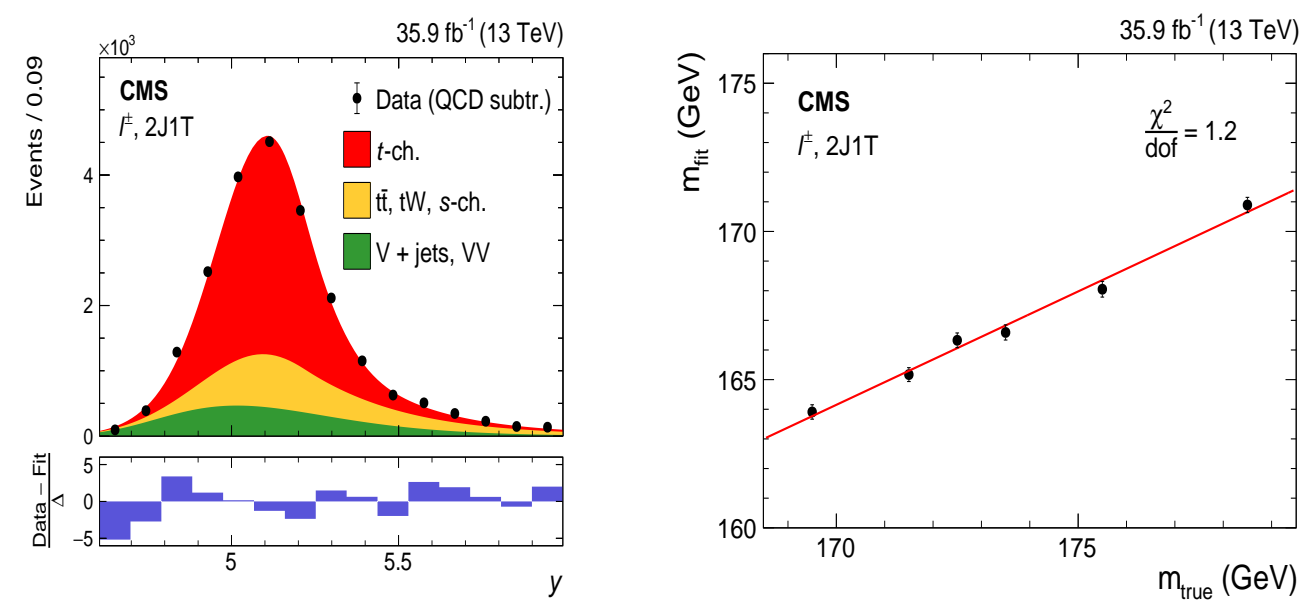

Figure 3: Postfit distribution of $y$ (left) and linearity of fitted vs. true mass (right).

\section{Summary}

The simultaneous fit in the electron and muon final state improves the overall signal yield in this analysis and helps in reducing the statistical uncertainty component. The multivariate analysis discriminator and the nuisance parameters in the maximum likelihood fit manage the background contamination to a level so that the impact of dominant sources such as JES can be brought undercontrol. These improved strategy adopted in this measurement is responsible for reducing the overall uncertainty in the measured top quark mass $m_{\mathrm{t}}=172.13 \pm 0.32$ (stat + prof $)_{-0.70}^{+0.69}$ (syst) GeV [2]. This result has a relative improvement of $29 \%$ in precision compared to the previous CMS measurement [4] in events with the same topology. The top quark and antiquark masses are also determined separately using the lepton charge in the final state. The mass ratio and difference are determined to be $0.9952_{-0.0104}^{+0.0079}$ and $0.83_{-1.35}^{+1.79} \mathrm{GeV}$, respectively. The mass difference value agrees with the previous CMS[3] measurement based on $t \bar{t}$ events [1]. These results are consistent with charge-parity-time invariance.

\section{References}

[1] Serguei Chatrchyan et al. Measurement of the mass difference between top quark and antiquark in pp collisions at $\sqrt{s}=8 \mathrm{TeV}$. Phys. Lett. B, 770:50, 2017. arXiv:1610.09551, doi: $10.1016 / j$.physletb. 2017.04.028.

[2] CMS Collaboration. Measurement of the top quark mass using events with a single reconstructed top quark in pp collisions at $\sqrt{s}=13 \mathrm{TeV}$. ArXiv e-prints, 2021. arXiv:2108 10407.

[3] CMS Collaboration. The CMS experiment at the CERN LHC. JINST, 3 (2008) S08004. doi : $10.1088 / 1748-0221 / 3 / 08 / 508004$.

[4] Albert M Sirunyan et al. Measurement of the top quark mass using single top quark events in proton-proton collisions at $\sqrt{s}=8 \mathrm{TeV}$. Eur. Phys. J., C77:354, 2017. arXiv: 1703.02530, doi:10.1140/epjc/s10052-017-4912-8. 